\title{
La mort de Papa Alexandre VI, segons un poema anònim italià de 1504
}

\author{
The death of Alexandre VI, acording to an anonymous italian poem \\ (1504)
}

Júlia Benavent

julia.benavent@uv.es

Universitat de València

Resum: Un poema en octaves sobre la mort d'Alexandre VI, inèdit i anònim, publicat a Itàlia, aporta noves dades sobre la literatura contra els Borja. En aquesta ocasió Alexandre VI és mostrat com un nigromàntic que no sap veure l'anunci de la seua mort.

Paraules clau: Alexandre VI, nigromància, segle XVI, Borja, poema

Abstract: A poem in octaves about the death of Alexandre VI, unpublished and anonymous, printed in Italy, provides new data on the literature against the Borja family. In this instance, the poem portrays Alexandre VI as a necromancer who cannot see the announcement of his own death.

Keywords: Alexandre VI, necromancy, 16th century, Borja, poem 


\section{Introducció}

El text que presentem és un imprès, sense data ni lloc, d'autor desconegut, del qual durant anys s'ha afirmat que fou publicat a Venècia cap a l'any 1508. Sobre aquest imprès Gerolamo d'Adda va publicar un article l'any 1875 a Archivio Storico Lombardo (D'Adda 1875: 15). Actualment el poema es pot llegir en una edició anagràfica a Guerre in ottava rima (Beer, Diamanti \& Ivaldi, 1989) que recull una bona mostra dels canti storici o relacions de fets ${ }^{1}$ sobre les guerres d'Itàlia.

Tot i que Gerolamo d'Adda els anomena canti storici, al llarg de l'article esmentat diu que aquest tipus de poesia popular, cantada o recitada en veu alta pels carrers i places a la Lombardia des del segle XVII, era coneguda com a bosina i d'ella en derivava la bosinaa o bosinada, ${ }^{2}$ és a dir, l'orígen de les primeres gacetes de notícies. Eren les ludibria ventis dels quals parla Virgili al cant VI de l'Eneida, notícies que corrien per tot arreu. Desconeixem perquè ha estat poc estudiada aquesta matèria, $\mathrm{i}$ per això no és possible de determinar quin tiratge tenia la publicació d'aquests fulls volants, com es distribuien i quins n'eren els autors i lectors. Per les característiques físiques de la publicació i la pressa en la seua divulgació, així com l'anonimat habitual, sabem que es tractava d'un text crític amb el personatge, en aquest cas Alexandre VI, que movia la burla dels ciutadans, aficionats a les pasquinades, i que tenien una vida molt curta, si atenem als pocs testimonis que es conserven a les bibliotèques públiques o privades.

Gerolamo d'Adda atribuia l'obra a l'autor Eustachio da Udine i proposava la data de 1508. El seu testimoni era un text de procedència privada, del qual no en citava el posseïdor que li l'havia facilitada. Els editors de Guerre d'Italia varen localitzar un testimoni a la Fondazione Giorgio Cini a Venècia, el tipògraf del qual fou identificat com a Giovanni Giacomo Risi, ${ }^{3}$ que habitava a Milà i que era actiu entre 1493 i 1519. A més, la data de la publicació fou establerta en 1504, més pròxima a la mort del papa Alexandre VI, esdevinguda a Roma en el mes d'agost de 1503, cap a meitat del mes.

La composició consta de 34 octaves que narren la vida del papa Alexandre VI, des del seu naixement a Xàtiva (Zatival la quale è trenta milia da lontano/ da la cità Valenza nominata), els esdeveniments dels darrers anys del seu regnat, fins a la mort i la posterior creació de Juli II que va ocupar el soli pontifical el dia 18 d'octubre de 1503. Narra també en la darrera octava, sota la forma d'un lament, la

1 La natura sobre el gènere d'aquestes composicions no està encara resolta. Gerolamo d'Adda els anomena canti storici i els editors dels 4 volums de les Guerre d'Italia els defineixen com a poemes en octava rima. Noves opinions consideren que en realitat, en vers o en prosa, aquests són els primers testimonis de la divulgació de notícies, que varen tenir una molt ampla difusió amb la invenció de la imprempta. L'autora d'aquest article ha publicat anteriorment altres textos de natura semblant (Benavent 2009 i 2011).

2 La bosina, bosinaa, o bosinada era una composició popular en vers, escrita en milanés, recitada per cantaires, de tema satíric. La primera de la qual es té notícia data de la meitat del segle XVII.

3 Sobre l'editor Risi, vegeu Borsa (1980), Ascarelli \& Menato (1989) i Sandal (1977-1981) Vegeu, encara, Short-title catalogue... (1986) i el lloc web de l'Istituto Centrale per il Catalogo Unico delle biblioteche italiane e per le informazioni bibliografiche - ICCU. Edit 16 http://edit16.iccu.sbn.it/web iccu/ihome.htm [consultat el dia 31 de desembre 2016]

SCRIPTA, Revista internacional de literatura i cultura medieval i moderna, núm. 9 / juny 2017 / pp. 273-285

ISSN: 2340 - 4841 doi:10.7203/SCRIPTA.9.10347 
caiguda del Duc Valentinès, Cèsar Borja, mort el 1507. Aquest darrer fet fou amb seguretat la causa de la datació de Gerolamo d'Adda en 1508, però era habitual que les composicions continuaren publicant-se molt desprès dels fets, aixì trobem reimpressions, o reescritures, molts anys desprès d'haver-se produit els fets que s'hi narren. En aquest sentit, la notícia es prorrogava invaint el camp de la llegenda.

Tanmateix hi ha una referència al text de la mort de Papa Alexandre VI que ens sobta especialment. Un gravat del frontispici fa referència a un mag, de front al lector, que assenyala amb la seua mà esquerra el cel amb la lluna i les estreles. Amb l'altra mà té un globus terraqui o una esfera, molt indefinida. És al camp, però a la mà dreta del lector es veu un llibre obert sobre una taula. L'altre gravat representa la Mare de Déu amb Jesús infant de peu sobre els genolls de sa mare, que acepta la reverència d'una dona agenollada davant d'ells. Al fons es veu un arbre i unes cases, a mà esquerra del lector, com una adoració. Els caràcters tipogràfics de la impressió són gòtics i les octaves estan distribuides en dues columnes. És en $4^{\text {rt }}$, dos folis, 2 columnes, 2 xilografies i la caplletra adornada.

Tota la història mou de la forta ambició de Roderic de Borja per tenir el di Piero glorioso manto. Un dia Roderic Borja entra en una cambra on hi havia un llibre de nigromància, i invoca un dimoni, anomenat Mamone. Aquest nom és utilitzat al Nou Testament per a personificar el profit i l'avarícia. Invocava, doncs, el dimoni de l'avarícia, del profit, de les riqueses. Segueix una part dialogada que contribuïa a la dramatització de la història entre el dimoni i el cardenal Borja, que li demana 19 anys de regnat a canvi del qual li lliuraria la seua ànima, i conclou amb l'anunci del dimoni sobre la duració del Papat: Undici octo Papa tu sarai. El cardenal Borja entén que eren onze anys més altres vuit, és a dir, els 19 anys que li havia demanat:

Se diecenove anni fai che papa sia

l'anima el corpo mio in toa balia (octava 8)

L'esment a l'octava 11 d'una profecia de san Cataldo (Casinelli, 1717), bisbe de Taranto, en la Puglia, que pertanyia al regne de Nàpols, ens fa pensar que la història de la mort prové de les terres del Sud, cosa que no contradiu que haja estat impresa a Milà. La profecia de san Cataldo deia que quan arribaria el bou a Itàlia, hi hauria guerres i desgràcies. San Cataldo és un sant d'orígens irlandesos, bisbe de Taranto, que fou empresonat per haver estat acusat de practicar la màgia. Al seu sarcòfag, descobert 10 segles desprès de la seua mort, s'hi trobà l'any 1071 un llibre de profecies, ben conegut de l'autor anònim del poema sobre la mort d'Alexandre VI, que recorda el gravat del mag que esmentàvem abans.

L'ambició del fill Cesar Borja, que necessitava diners per a fer les guerres de la Romanya, els portà a fer el sopar famós de l'enverinament, amb l'assistència dels rics cardenals. S'ha dubtat molt sobre els convidats al sopar. Per cartes escrites aquells dies i els successius dels ambaixadors de 
Ferrara i de Venècia se sap que el papa estava malalt des de setmanes abans i que els símptomes de l'enverinament podien haver estat causats per unes febres terçanes que afectaven aquell estiu la ciutat de Roma i que provocava efectes semblants a la mort per verí. Malgrat els testimonis d'Alessandro Sardi, contemporani de Guicciardini i de Giovio, que escrivia a Ferrara, on seria puntualment informada la filla Lucrècia, el sopar del verí resta una referència obligada de la família Borja. Voltaire a Essai sur les moeurs ${ }^{4}$ aduïa molts arguments contra l'enverinament. Pensava que una distracció del credencer era una raó poc versemblant per qui estava habituat a aquestes malifetes, a més Cèsar no hauria estat perdonat pels cardenals supervivents, i seguia preguntant-se com era possible que tots es posaren d'acord sobre la manera de contar la història, el nom del verí, canterella, i concloïa que es fàcil inventar una història quan s'acusa algú d'actes des de temps enrere. Cert o no, el text que avui presentem barreja la mort del Papa, la ficció de la nigromància, la profecia de san Cataldo, la cantarella i Voltaire, cosa que cada dia sembla més familiar quan es tracta dels Borja, des de fa cinc segles ja. Les darreres octaves aclarien la broma del dimoni Mamone, que havia enganyat el Papa fent-li creure que regnaria 19 anys, però el dimoni sumava als onze anys huit dies.

\title{
2. Edició del text
}

Els criteris adoptats en la següent edició són molt respectuosos amb l'original per tal de garantir l'estat de la llengua italiana amb els seus trets característics. Les intervencions atenen a la separació de les paraules, l'ús de l'apòstrof, l'accentuació, la distinció entre $u$ i $v$, la regularització de la puntuació, que ha exigit la revisió de l'ús de majúscules i minúscules. Ha estat també destacat amb cometes l'estil directe les parts de les dialogades.

\section{Questa si è la morte de papa Alixandro Sesto}

Venezia, Fondazione Cini: 1091

1

\begin{abstract}
Clemente Dio, che recuperasti col tuo sangue l'umana natura im prima il sancto verbo predicasti e fusti morto dala gente dura, a li nostri peccati non guardasti, per te fu salva ogni creatura: prestami gratia che io canta in gesto la morte del papa Alexandro sesto.
\end{abstract}

4 Voltaire, Essai sur les moeurs 110, 1756 (Versaille, 1994). 
Júlia Benavent. La mort de Papa Alexandre VI, segons un poema anònim italià de 1504

2

Nel mille cinquecento e tri anni morì Alexandro, el qual fu pastore e fu molesto a molti christiani, li quali sun destructi con furore, con crudeltà, acerbi torti e sani perché il cielo li dava favore. Undeci anni stete nel papato e octo giorni como honorato ${ }^{5}$.

3

Fu il papa Alexandro valenciano da una terra Zativa chiamata, la quale è trenta milia da lontano da la cità Valenza nominata; fizuolo fu d'un citadino soprano, ebe soa persona ingenerata.

Papa Calisto el fece cardinale, ch'era suo nepote principale.

4

Essendo Alexandro in etade fra tutti gli altri era honorato, cresse costui in grande facultate, femine e maschi hebbe generato, mostrava haver in sè gran caritade e ebbe a Roma triumphante stato, haveva corte come rechedia, sì come a su pare convenia.

Il papa Paulo di vita fu casso il concistoro fece il papa Sixto, non se vedeva Alexandro lasso, ma tutte l'hore stava ben provisto. Da ogni tempo studiava il passo, solo per esser vicario de Christo, essendo Sixto de vita passato, fu dato a Inocentio il papato.

5 horonato a l'original.

SCRIPTA, Revista internacional de literatura i cultura medieval i moderna, núm. 9 / juny 2017 / pp. $273-285$ ISSN: 2340 - 4841 doi:10.7203/SCRIPTA.9.10347 
Júlia Benavent. La mort de Papa Alexandre VI, segons un poema anònim italià de 1504

6

Era questo Alexandro sì bramoso

haver di Piero il glorioso manto

et era tanto al fin desideroso

sì come parla dice questo canto.

Morì papa Nocentio virtuoso,

el concistore sa raduna in tanto

per far un papa che a Roma governasse,

aciò che al fin iusticia regnasse.

7

Costui haveva al fin deliberato

da esser fato papa dei christiani, e presto in una camera fu intrato uno libro haveva con inganni e per nigromantia ha chiamato uno dimoni odi quelli tiranni, e quel demonio vene tutto ardito "al to comando sarai ubedito".

8

Questo dimonio se chiamò Mamona, disse Alexandro: "Voglio el papato, integramente voglio la corona".

Dise el dimonio:"Questo sarà fato, incoronato sarà tua persona" e l'un con l'altro refirmò il pacto: "Se diecenove anni fai che papa sia l'anima el corpo mio in toa balia".

9

Alhora il dimonio parlò scuro:

"Undici octo papa tu sarai, di questo certo io ti fo securo che a to piacere signorezarai". Alexandro non intese il caso duro. $\mathrm{O}$ come inganato tu sarai, questi octo giorni che de più scampato credeva tanti anni fusse il pato.

10

Essendo in concistoro di cardinali, el manto Alixandro li fu dato.

Monsignor Aschaneo e altri principali

SCRIPTA, Revista internacional de literatura i cultura medieval i moderna, núm. 9 / juny 2017 / pp. 273-285 ISSN: 2340 - 4841 doi:10.7203/SCRIPTA.9.10347 
Júlia Benavent. La mort de Papa Alexandre VI, segons un poema anònim italià de 1504

S'an però in Vincula dotato.

$\mathrm{Da}$ alhora in qua Italia è stata male e ia questo fu per il nostro peccato, che Dio ce il donò per penitentia e inde Dio iudicio e sententia.

11

E questo profetizò san Cataldo quando el bo in Italia parirebe e che christiani combataran saldo e che terribil guerra poi sarebbe e per l'oribel fogo sparto e caldo che molte gente se ociderebe solo per Italia e per il so dominio e che il terreno se farebe minio.

12

Essendo Alexandro stabilito deliberò voler signoregiare le terre de la Giesa, il santo sito e de molti signori se scaciare. Vedevasi la signoria a mal partito, ma non doveva il papa questo fare, doveva fare como iusto e inmenso pigliar da ciascun qualche incenso.

13

Quando il re di Franza vien a sdigno col signor Ludovico de Milano e che fo discacia' for dil so regno, el papa Alexandro se fe strano, dicendo: "Il mio fiolo sarà degno ie sarà Imperator d'ogni christiani o son papa se lui sia imperatore c' al mondo non sarà magior signore.

14

Diceva Alexandro: "Son disposto che vol la santa Giesa difensare e Valentin alhora hebbe risposto: "La mia fama al cielo voglio alzare, or sapi pare che non starò ascosto, che tutto il mondo i farò tremare". E molta gente in ponto metia, paiso asai conquistato havia.

SCRIPTA, Revista internacional de literatura i cultura medieval i moderna, núm. 9 / juny 2017 / pp. 273-285 ISSN: 2340 - 4841 doi:10.7203/SCRIPTA.9.10347 
Júlia Benavent. La mort de Papa Alexandre VI, segons un poema anònim italià de 1504

15

Achade che 1 duca Valentino

voleva ruper guerra a senesi.

Era superbo più che mai Tarquino

deliberò de star ale contesi,

sì come volse fortuna e destino.

Fo necesaro abandonar l'impresi,

auditore, se ascoltariti

tutto il conveniente intenderiti. // (f.2r)

16

Ma prima che il duca se movise

al padre suo domandò consilio.

Il papa alhora a Valentin dise:

"Tu meterai to gento in gran scompilio".

Respose Valentin: "Se provedise

i poterei campar d'ogni perilio.

Tuta mia gente mi vol seguitare,

ma io non ho il modo di pagare".

17

Rispose il papa: "Nostri cardinali

sì se prestarà argento e oro,

quelli che de nostra corte principali,

questi te campare d'ogni martoro,

fa che la tua fama in alto sale,

e dapoi ciascadun farai ristoro.

E per volere io voglie satisfare, presto li cardinali fe chiamare.

18

"O Monsignori, alti e reveren,

a mi fa de bisogno el vostro aiuto,

voi sui generosi e clementi,

viati humanità senza tributo".

Rispose li cardinali: "Stiam contenti,

il tuo domandar sarà compiuto".

Rispose Valentin, con belli sembianti:

"Ora imprestatime trenta milia bisanti".

SCRIPTA, Revista internacional de literatura i cultura medieval i moderna, núm. 9 / juny 2017 / pp. 273-285

ISSN: 2340 - 4841 doi:10.7203/SCRIPTA.9.10347 
Júlia Benavent. La mort de Papa Alexandre VI, segons un poema anònim italià de 1504

19

Rispose li cardinali: "Non havemo

e vel faremo vedere e tochare".

Dicivia Valentin: "Come faremo?"

Li cardinali s'ebbe a scombiatare".

Dise Alexandro: "Nu provederemo se al mio modo tu vorai fare, uno bon disnare l'invidaremo. Il tosico a termine li daremo".

20

E fe venire quatro fiaschi de vino, del megliore che si possa trovare, in uno sol fiasco misse quel venino e poi li cardinali se convitare diciva in ver del padre Valentino:

"Questo è un tradimento che non pare

Questi veneni ch'abiam fat a vicio sopra de noi potre tornar iudicio".

21

Dise Alexandro: "I son nostri inimici, col tempo ce farà mal capitare. Tu sei sopra li signori così felici. Ora ti spaza senza più indusiare e chiamar li faremo como amici". E si li fece far un bel disnare e for metuti a tavola setati li fia $<$ s $>$ chi al credencer furno signati"'.

22

Il fiascho che haveva tosicato di differenciato se li consignò. Dice il papa: "Tu l'harai portato quando alhora te'l domandarò". Rispose el credencier:"El serà fato, ma insiema con li altri lo lasso". Sentato i cardinali ognun sublimo el vin atosicato fu il primo.

El credencer de questo non sapeva del tradimento quale se usava, el papa in gran festa si staseva 
Júlia Benavent. La mort de Papa Alexandre VI, segons un poema anònim italià de 1504

con li cardinali in zolia triunphava.

Come il papa disnato haveva,

el vin al credencer domandava.

Rispose il credencer con reverentia,

il fo il primo vin che ven a mensa.

24

Lixandro dala mensa fo levato, alhora se scoperse el tradimento. Ognuno se vedeva imbratato, il tosico li dava impedimento. Lixandro in una camera fo intrato, maladicendo tal proponimento. Longo desteso se geta sul leto, vene la morte inante al so conspeto.

Dice la morte: Alexandro mio or voli fati toi con Dio conzare, per te me a mandato il sumo Idio, or voli a penitenta ritornare, tu sai che "l to voier è stato rio. Tu avesti tutto il mondo a dominare. In prima che la morte li disparse, alhora quel $\operatorname{dim}<\mathrm{o}>$ nio si li aparse.// (f.2v)

26

Diceva il demonio al pastore:

"Voiu la mia promessa observare". Rispose Alexandro con dolore: "Non za che tu m'avuto anganare". Rispose il dimonio: "Il ponto e l'ore t'o voluto tuto quanto sotisfare". Dice il papa: "Tu m'ai fato inganni, il nostro pato fu per disnone anni".

27

Dice il dimonio: "Abi pacientia che non parlai a mesi ne a semani, undeci octo fo nostra sententia, sopra l'inganator verà l'inganni.

Tu ai fato a tanti violentia, O quanti ne ai mesi in scorni e dani, a torto, con viltà senza ragioni e che hai incritato dannatione". 
Júlia Benavent. La mort de Papa Alexandre VI, segons un poema anònim italià de 1504

28

Risposa Alexandro: "Ora mi pento

a Jesù i domando perdonanza, de ogni tristo falso falimento,

O Dio, non guardar a mia ignoranza".

Rispose il dimonio con spavento:

"Tu sei ormai sul crol de la bilanza,

convien tu sia pagato secondo el merto

Idio al dimonio t'a offerto".

29

Diceva Alexandro: "Salvatore

prego che 'l mio peccato me perdoni

a ti me rendo con perfecto core,

fa che al fin la gloria tu me doni".

Rispose il demonio inganatore

tu sarai posti nel infernal toni,

sì che Idio non te po' salvare,

d'endar in paradiso non te "l pensare".

30

Haveva Alexandro contritione

del gran peccato ch'aveva commesso.

Rispose alhora al dimonio felone,

convien che a te l'inferno sia conceso.

Tu credi haver da Dio salvatione

e da po 'li mostrò il so processo,

del gran peccato doloroso e forte

in questo mezo vien l'aspra morte.

31

Sì che Alexandro de vita fu caso.

El duca Valentin fa gran lamento:

"Tuto el mio stato andarà a fracaso, piangeva forte con dolor e stento. Sarai coperto sotto un duro sasso.

O quanto me ritrovi mai contento

da poi che sei di vita pasato

sarà a me contra il cielo rivoltato.

32

"Non era necessario, o padre mio, atosicar nesun de nostra corte.

Questo è stato iudicio de Dio,

SCRIPTA, Revista internacional de literatura i cultura medieval i moderna, núm. 9 / juny 2017 / pp. 273-285

ISSN: 2340 - 4841 doi:10.7203/SCRIPTA.9.10347 
Júlia Benavent. La mort de Papa Alexandre VI, segons un poema anònim italià de 1504

onde che tua vita è stata corte, revoltarase mie provincie a io e da poi se lamenta de la morte. Tu m’a gonto in questi miseri anni sopra l'ingannator torna l'inganni"'.

33

Diceva Valentin: "O morte cruda, sen padre mio viveva doi anni piangendo forte il fronte li iuda, O quanto è stato li mei penseri vani restava la mia persona nuda, e mai più spero de uscir d'afanni haveva fato guerra contumace volivi meter tuto el mondo in pace".

34

Essendo morto Alexandro pastor, el ducha Valentin fu destruto il segio suo di tanto valore e facilmente fu privato del tuto, el stato che acquistò senza rumore e così senza rumone l'a perduto ha perso ogni vitoria el magno sito al vostro onore questo canto è finito.

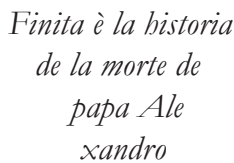




\section{Bibliografia}

Ascarelli, Fernanda \& Menato, Marco (1989) La tipografia del '500 in Italia, Firenze, Olschki.

Beer, Marina \& Diamanti, Donatella \& Ivaldi, Cristina (eds.), (1989), Guerre in ottava rima, Istituto di studi rinascimentali Ferrara, Modena.

Benavent, Júlia (2009) "Poemes inèdits sobre Cèsar Borja", Revista Borja. Actes del 2 Simposi Internacional sobre els Borja, núm. 2, pp. 13-20.

---- (2011) "Edició dels poemes sobre Cèsar Borja”, Revista Borja, núm. 3, pp. 113-189.

Borsa, Gedeon (1980), Clavis typographorum librariorumque Italiae 1465-1600, Aureliae Aquensis, aedibus Valentini Koerner. 2 vols.

Casinelli, Cataldo Antonio (1717) Vita e memorie di san Cataldo, Nàpols.

D'Adda, Gerolamo (1875) "Canti storici popolari italiani”, Archivio Storico Italiano, X.

Sandal, Ennio (ed.) (1977-1981) Editori e tipografi a Milano nel Cinquecento, Baden Baden, Valentin Koerner, 1977-1981. 3 vols.

Short-title catalogue of books printed in Italy and of Italian books printed in other countries from 1465 to 1600 now in the British Museum. London, The British Library Board, 1986

Versaille, André (1994) Voltaire, Essai sur les moeurs 110, 1756. Dictionnaire de la pensée de Voltaire par lui même. Bruxelles. 